\title{
Bioanalysis
}

\section{9th GCC Closed Forum: CAPA in regulated bioanalysis; method robustness, biosimilars, preclinical method validation, endogenous biomarkers, whole blood stability, regulatory audit experiences and electronic laboratory notebooks}

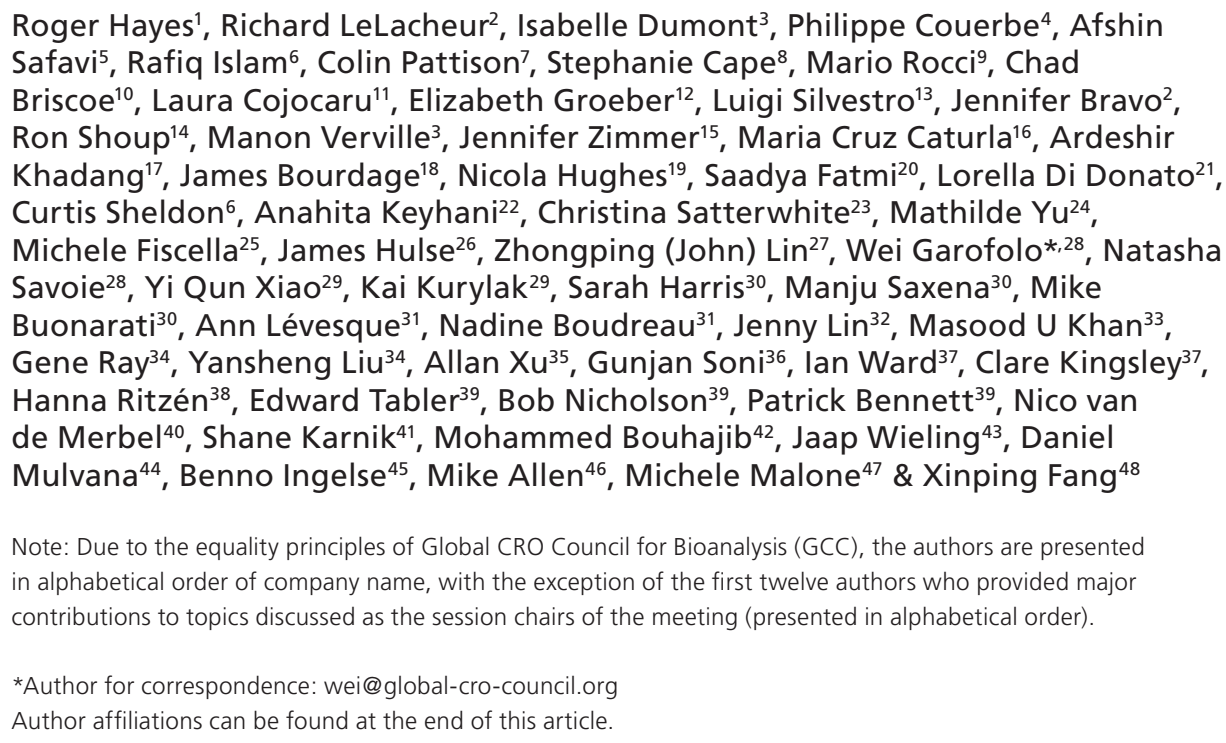

9th GCC Closed Forum, Miami, FL, USA, 13 April 2015

The 9th GCC Closed Forum was held just prior to the 2015 Workshop on Recent Issues in Bioanalysis (WRIB) in Miami, FL, USA on 13 April 2015. In attendance were 58 seniorlevel participants, from eight countries, representing 38 CRO companies offering bioanalytical services. The objective of this meeting was for CRO bioanalytical representatives to meet and discuss scientific and regulatory issues specific to bioanalysis. The issues selected at this year's closed forum include CAPA, biosimilars, preclinical method validation, endogenous biomarkers, whole blood stability, and ELNs. A summary of the industry's best practices and the conclusions from the discussion of these topics is included in this meeting report.

First draft submitted: 8 January 2016; Accepted for publication: 26 January 2016; Published online: 26 February 2016

Keywords: biomarker $\bullet$ biosimilar $\bullet$ blood partitioning

The 9th Global CRO Council in Bioanalysis (GCC) Closed Forum took place on 13 April 2015, preceding the 9th Workshop on Recent Issues in Bioanalysis in Miami, FL, USA. The GCC was founded in 2010 to allow senior management level CRO rep-

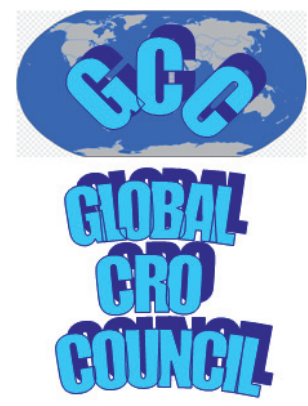

FUTURE SCIENCE part of 
resentatives to share experiences and opinions related to scientific and regulatory issues in bioanalysis [1]. Eight prior closed forums have periodically occurred since the founding of the GCC, typically associated with other major bioanalytical conferences, to discuss a variety of topics and issues of particular interest to bioanalytical CROs. Conference reports describe the discussions, allowing a glimpse into the opinions and challenges faced by this subsection of the industry [2-6]. Additionally, several White Papers have been published by the GCC on topics of particular widespread interest [7-12]. These White Papers provide recommendations that are meant to be applicable to the global bioanalytical community.

Roger Hayes, chair of the 9th GCC, began the meeting in traditional fashion, with the official admonition statement [1], which instructed participants on the guidelines to follow during the discussions. Following this, all participants introduced themselves and then this year's discussion topics were presented. The eight topics for the 9th GCC Closed Forum were as follows:

- The role of CAPA in regulated bioanalysis.

- Method robustness in regulated bioanalysis.

- Best practices for supporting biosimilar studies using ligand binding methods.

- Status of method validation requirements in the preclinical space.

- Best practices for measuring endogenous biomarkers beyond prior GCC recommendations.

- Whole blood stability and blood partitioning.

- Regulatory audit experiences.

- Electronic laboratory notebooks and LIMS systems.

\section{Discussion topics}

The role of CAPA in regulated bioanalysis

A robust Corrective Action and Preventive Action (CAPA) program is required in a Chemistry, Manufacturing and Controls (CMC)/Good Manufacturing Practices (GMP) environment in accordance with the Q10 GMP guidance document [13]. The core value of such a program is to determine the root cause of any quality issue resulting from an investigation into a nonconformance, complaint or other undesirable situation. Once determined, the root cause is addressed by a two-pronged approach. The first is the corrective action, which is implemented to address the cause of a detected issue. The second is the preventive action, which is implemented to prevent the detected issue from recurring.
In the context of manufacturing drug products, CMC/GMP principles outlined in the Q10 document are applied to ensure that drugs are consistently produced and controlled in such a way as to meet the quality standards appropriate for their intended use, as required by the marketing authority. The CAPA program, when implemented properly, should result in a greater understanding of the product and processes and, therefore, their improvement. Part of the regulatory requirements of the program includes complete and thorough documentation of the entire process involved in a CAPA, which typically includes seven basic steps:

- The identification of the problem/potential problem, nonconformity or incident;

- An evaluation of the magnitude of the problem and potential impact on the company;

- The development of an investigation procedure with assignments of responsibility;

- Performing a thorough (root cause) analysis of the problem with appropriate documentation;

- Creating an action plan listing all the tasks that must be completed to correct and prevent the problem from recurring;

- The implementation of the plan;

- A thorough follow-up with verification of the completion of all tasks, and an assessment of the appropriateness and effectiveness of the actions taken.

Based on feedback from sponsors to CROs, there appears to be an expectation that GLP/regulated bioanalytical laboratories meet these GMP requirements despite the fact that they are out of scope for GMP regulations. There is a fundamental difference between GMP and GLP, however, which gives pause to the CROs when trying to decide how best to fulfill the expectations of their clients. At a high level, GMP is concerned with the integrity and quality of a manufacturing product intended for human use, whereas GLP protects the integrity and quality of laboratory data used to support a product application.

There are undisputed benefits to implementing a CAPA program in bioanalysis, including better control of processes and methods, better understanding of issues, a decrease in the risks of failures and an increase in data accuracy and reliability. Conversely, there are potential drawbacks if a CAPA program is implemented inappropriately. These include additional work and costs as well as inadequate investigations leading to a waste of resources (human, time, finan- 
cial) with no significant added value to the generated bioanalytical data.

In an effort to understand how and if CROs are electing to implement CAPA programs, a survey was sent to CRO member companies asking to share their approaches and philosophies. A majority of the respondents indicated that a CAPA program is pertinent to regulated bioanalysis, but should be judiciously applied and is not intended for all quality issues. The results indicate that most respondents believe that the quality issues that should be addressed by a CAPA program are those that are recurrent or systemic in nature, not isolated, or those that have significant impact on the bioanalytical data being generated.

The discussion with the attendees seemed to support these survey results. There was agreement that while the idea of a CAPA program is a good one, the extent of a GMP-like program is not required, and the investigation of quality issues should be outlined in a written procedure or Standard Operating Procedure (SOP). There is an assumption in GMP that methods and processes are designed to never fail; however, in GLP, tolerance limits are built into the methods, and failures do not have the same impact. For example, the instability of an analyte during a sample extraction procedure requires a method adjustment, not necessarily a full investigation into the cause of the stability issue. It was noted that most attendees perform trending at their companies in order to decide whether a CAPA approach to the remediation of quality issues is appropriate. Some examples of issues that may require a CAPA approach can include significant documentation lapses, lost samples or chronic contamination issues.

In conclusion, in a regulated bioanalytical CRO environment, CAPA elements should be thoughtfully applied by considering a balance between expected benefits, resources needed and potential risks for the organization. The CAPA approach should be tailored and adapted to fit the area of regulated bioanalysis. Each quality issue should be evaluated on a case-bycase basis prior to applying CAPA; the impact, frequency and significance of the issue should drive the decision to implement CAPA (i.e., risk-based CAPA).

\section{Method robustness in regulated bioanalysis}

Another topic discussed was that of 'GMP creep' into regulated bioanalysis and the expectation of how method robustness should be evaluated. The ICH Q2(R1) guideline for validation of GMP analytical methods [14] refers to small but deliberate variations in the method that could impact the reliability of the results. This is further referenced in the 2015 US FDA guidance document [15], which calls for a systematic approach for method robustness. However, the notion of evaluating the variations in the bioanalytical method is not excluded from the international Bioanalytical Method Validation guidelines [16-19]. All four documents speak of performing precision and accuracy evaluations on different days. The FDA guidance further recommends performing the tests with different analysts, equipment, reagents and laboratories. The EMA recommends testing at least one run in a size equivalent to the anticipated study sample runs. However, GMP validation parameters suggest showing method reliability with many more variables, including $\mathrm{pH}$ of the mobile phase, temperature, flow rate, etc.

CRO member companies were surveyed to determine their approach to method robustness. The majority of respondents indicated that method robustness in regulated bioanalysis should be evaluated and so there was further inquiry into the variables that were tested. Most believed that validation runs should be performed on different days. Different analysts would only be required when the size of the study and the intended number of analysts used during the application of the method warrants it (i.e., such an assessment may not be necessary or applicable for small studies or when only a single analyst is involved). The use of different columns should be evaluated only if the need for multiple columns is anticipated (e.g., analysis of large numbers of study samples and use of multiple chromatographic systems). If the use of different lots of critical reagents is expected in ligand binding assays (LBA) or hybrid LBA/LC-MS methods, then this variable may need to be tested. There was no consensus on the evaluation of run size. Some suggested that only the largest anticipated run size should be tested, while others believed that because variable run sizes were inevitable during method validation, any additional tests were not required. Still others believed it depended on how many study samples were anticipated during the use of the method. Finally, respondents were asked if any other variables should be evaluated. Many mentioned that there might be other method specific parameters that may need to be investigated, but that this was method dependent.

In conclusion, method robustness testing in bioanalysis confirms that method performance will not be affected by variations in some parameters. The parameters to include in the testing should take into account the assay and the conditions expected during sample analysis and should be tiered based on the purpose of the method.

\section{Best practices for supporting biosimilar studies using ligand binding methods}

Regulatory agencies have provided guidance documents relevant to biosimilars, since these medicinal 
products have been submitted with increasing frequency $[20,21]$. However, despite these guidance documents, the relative infancy of these types of submissions has resulted in numerous, inconsistent approaches to meeting the recommendations requested by the agencies for PK assays, immunogenicity assays and critical reagent characterization. In order to determine how the industry is applying these recommendations and guidances, survey questions were sent to the GCC member companies. The first set of questions inquired after PK assays used to support biosimilar studies. The results for this type of assay indicate that the majority of respondents use one assay to measure both the innovator and the biosimilar drugs. This is based on the assumption that both drugs are identical, which is often not the case. Therefore, care should be exercised to ensure that this is the best approach. Should two assays be needed, samples should be run with both assays and bridging assays are needed to be able to appropriately evaluate the results. Calibrators are typically prepared with the biosimilar; however, QC samples during both method validation and sample analysis are prepared with both the biosimilar and the innovator test articles. Method comparability is demonstrated by using back-calculated concentrations (interpolated from one curve) of one set of QCs against the other with acceptance criteria of $\pm 20 \%$. Differences in concentrations between biosimilars and reference (innovator) products need to be investigated. It was noted in the discussions that correction factors may not be considered acceptable by the regulatory agencies.

Multiple lots of both reference and biosimilar drugs are typically evaluated during method development. Key reagent characterization, including purity, purification method and formulation, is performed for both the biosimilar and the innovator; however, the binding characteristics of the capture/detection reagents to the biosimilar and reference product are not typically evaluated. Relative potency testing is performed only if there are differences between the protein determination methods.

Regarding immunogenicity assays, respondents were evenly divided on the use of one or two assays. For those who use one assay, respondents use the biosimilar as the drug and use only one positive control. In the one assay format, evaluating the confirmatory cutpoint by using only the biosimilar is common practice for the majority of respondents. For those that use two assays, the acceptance criterion for proving biosimilarity is results within 2-3-fold for sensitivity, cutpoint and drug tolerance. For neutralization assays for biosimilars, cell-based neutralizing antibody assays are typically selected although ligand binding assays can be used.
Status of method validation requirements in the preclinical space

The GCC membership was surveyed regarding the different approaches to bioanalytical method validation used under differing circumstances, and the confusion surrounding the use of the popular term 'GLP validation.' GLP regulations detail quality systems, controls and documentation and do not specifically address bioanalytical method validation requirements. These are defined in guidance documents and regulations [16-20].

Therefore, initial survey questions attempted to determine how industry labeled GLP and GCP bioanalytical work. The majority of respondents use the term 'Regulated Bioanalysis,' and less than a third distinguish between clinical and preclinical bioanalytical validation. It should be noted that approximately $60 \%$ of respondents stated that they performed more than $75 \%$ clinical work. Of this group, less than $25 \%$ of the clinical work were bioequivalence (BE) studies. The attendees discussed at length whether GLP regulations should be used to support clinical studies, even though they fall out of scope. There was no consensus, although the use of Good Clinical Laboratory Practices [22] was suggested as an alternative approach. This may be limited by individual regulatory authorities' use of GCP inspections for laboratories supporting clinical trials, however.

For those that perform a variety of study types (clinical, preclinical, $\mathrm{BE}$, non-BE), the majority stated that validation testing is not tiered based on the type of study. Further, regulatory audit experience seems to demonstrate that auditors tend to audit against full GLP compliance, not using a tiered framework, although they do also accept this latter approach.

The use of a tiered approach to method validation was evaluated via the survey. The responses indicate that there are two situations that favor its use: 1) analytical challenges preclude it; and 2) the end use of the data does not require full validation. Attendees noted that sponsors are generally hesitant to use methods that have not undergone full validation. When a tiered validation is used, there is no consistent language used to describe it, and a variety of terms are employed; for example, partial validation, qualification, exploratory method. Although no consensus was reached at the closed forum, attendees did desire that harmonized language for these types of methods should be agreed upon. The use of tiered validation for preclinical studies seemed to be favorably accepted, but was much less acceptable when used for clinical studies.

Several scenarios were proposed to identify when respondents believe that using a tiered validation would be appropriate. A tiered approach was supported by the majority of respondents for at least the secondary 
study endpoint, if the situation warrants and regardless of human or animal species. One example would be if rare matrices were being assayed (e.g., tissues or cerebrospinal fluid). For common matrices, respondents were more comfortable using a fully validated method. Other potential situations for using a tiered approach would be for biomarkers or minor, inactive metabolites.

\section{Best practices for measuring endogenous biomarkers beyond prior GCC recommendations}

Down-regulated biomarkers are endogenous substrates that, upon treatment, result in lower concentrations. Therefore, potentially very low concentrations are expected during the quantification of these analytes, causing a particular challenge when selecting a suitable assay matrix and subsequently trying to meet selectivity, recovery and bias requirements during method validation. In order to determine industry best practices for the quantification of endogenous biomarkers beyond those already recommended by the GCC [23], a survey was sent to both LBA and LC-MS bioanalysts asking what types of matrices are used in their assays.

LBA bioanalysts were evenly divided over the use of authentic matrix or buffer matrix to prepare standards when the endogenous level of the biomarker is below the LLOQ. Conversely, when the endogenous level is very high, buffer matrix was most commonly selected, with a still significant number of respondents electing to use a surrogate matrix (i.e., from another species). It was specified that when anything other than authentic matrix is used, dilutional linearity and parallelism must be verified. For the preparation of validation QC samples when endogenous concentrations are low, bioanalysts overwhelmingly select authentic matrix, pooled matrix is preferred and then fortified with a commercially obtained recombinant biomarker or the high calibrator from the assay kit. When endogenous biomarker concentrations are high, the use of diluted authentic matrix was recommended, although there were differences on how the diluted matrix was prepared. These different methods included mixing various individual lots of authentic matrix to obtain the desired concentrations, diluting authentic matrix with buffer or diluting authentic matrix with depleted matrix. It was noted that respondents typically screen between 10 and 30 individual lots of matrix to find appropriate endogenous levels for their assays.

Different approaches are used by LC-MS bioanalysts. When endogenous biomarker concentrations are low, typically multiple lots of authentic matrix are screened and those with the lowest levels are selected and used for preparing standards. A significant number of respondents also use a surrogate matrix. Authentic matrix is then used to prepare validation QC samples. When very high endogenous biomarker concentrations are expected, standards are prepared with surrogate matrix. Validation QC samples are prepared with authentic matrix for mid and high QCs, and diluted or surrogate matrix is used for low and LLOQ QCs. Some options for surrogate matrix include phosphatebuffered saline; phosphate-buffered saline with bovine serum albumin or human serum albumin in order to take into account the protein content of authentic biological matrix and the increased solubility of hydrophobic compounds; synthetic matrices; or stripped matrix (note that carbon stripping may be variable from lot to lot, and affinity extraction is costly and time consuming). However, the use of a surrogate matrix has some specific issues that must be investigated, such as analyte solubility differences in synthetic aqueous matrix, partial insolubility or partial precipitation or adsorption of nonpolar analytes and analyte recovery differences in surrogate matrix. If authentic matrix is preferred, then it may be possible to use a surrogate analyte in the form of a stable isotope-labeled (SIL) analyte, assuming that the properties of the authentic analyte and SIL analyte are the same and the mass difference between the two is sufficient such that the quantification will not be affected. The internal standard would then be a differently labeled compound or an analog of the analyte. If an SIL analyte is selected, then a correction factor must be applied by comparing the response factors of both the authentic and SIL analyte. The correction factor can be determined by preparing two curves each using surrogate and authentic matrices, determining the response factors and then taking the ratio. Of the two approaches presented above, that is, surrogate analyte/authentic matrix versus authentic analyte/surrogate matrix, the comparison data using the former gave the best results. The effect of matrix variability should be investigated and stability must be done in the authentic matrix.

\section{Whole blood stability \& blood partitioning}

The Bioanalytical Method Validation guidelines and regulations [16-20] recommend that analyte stability should be considered from the time of sample collection through all handling until analysis. As a response to this recommendation, to address stability during the sample collection procedure, the industry began performing whole blood stability during method development or validation. The European Bioanalysis Forum provided recommendations in 2011 [24], reporting that many bioanalysts agree that plasma stability is an acceptable surrogate for blood stability in approximately $95 \%$ of the cases, with the exception of mol- 
ecules containing certain functional groups such as $N$-oxides or hydroxamic acids. They recommended analyzing whole blood using a qualified assay. That same year, the GCC recommended assessing whole blood stability using a validated plasma method [7], wherein time 0 samples were drawn and immediately centrifuged to harvest the plasma and compared with stability samples which were left at testing conditions prior to harvesting the plasma. However, any partitioning of the analyte into red blood cells, cell surface binding or platelet binding is not taken into account. One attendee recounted their experience of an FDAdirected audit for a product submission. The goal of the audit appeared to be to understand how the compound behaved in whole blood and hemolyzed plasma. However, when the question of whether there had been any regulatory feedback on the conduct of whole blood stability testing during routine audits was posed during a survey, the majority of respondents indicated that there had been none. The limited feedback that had been received during audits seemed to indicate that the agencies were satisfied with the generally accepted testing of harvested plasma after the blood had remained at ambient temperature for at least $2 \mathrm{~h}$.

The survey attempted to determine the performance of the whole blood stability evaluation by bioanalysts following the earlier GCC recommendations [7]. The majority of respondents routinely conduct sample collection stability testing, typically in support of regulated studies (i.e., GCP or GLP studies). A significant number only perform this test for BE studies, and only a small number perform this test in support of discovery studies. In general, respondents believe that conducting stability testing in whole blood adds scientific value beyond what is learned through routine plasma testing. The evaluation is typically performed by processing the blood sample to plasma prior to analysis and determining the relative concentration versus the control sample. Survey results were less conclusive when the respondents were asked if their test procedure allowed the analyte to reach partitioning equilibrium prior to conducting the experiment. The majority did allow for equilibration, and time 0 and test samples were kept at the same temperature. However, comments indicated that in some cases, partitioning is only evaluated as needed if an issue is detected. It was proposed to use a radiolabeled analyte to definitively determine the partition coefficient in order to properly demonstrate whole blood stability, but this option was largely deemed unnecessary.

If testing results indicated that a significant percentage of the analyte partitions into the red blood cells, most respondents would consider redeveloping the method using whole blood as the matrix, although a significant percentage would not necessarily do so; it would be evaluated on a case-by-case basis, typically by the sponsor. Finally, if instability in whole blood is demonstrated, there were a variety of opinions regarding the validation of the accompanying method. Many thought that the method could not be used in its current form. Others believed that the method could still be considered valid and used to support regulated studies while still others believed that it could only support nonregulated studies. It was clear, however, that the impact of the instability needs to be assessed and documented, and the resulting actions to mitigate the impact must be clearly indicated. Some potential actions include adjusting sample handling procedures to ensure stability by changing the temperature, shortening the processing time or using a stabilizer in the collection tubes.

In conclusion, the original recommendations provided by the GCC [7] were still supported and no new updates appear necessary.

\section{Regulatory audit experiences}

A discussion surrounding the regulatory experiences of attendees occurred, with a variety of findings being presented along with suggestions for compliance, when available.

- Test article characterization: the quality assurance (QA) unit failed to review the raw characterization data of the test article, and only assured that the certificate of analysis (provided by the sponsor) was available. Nor did the QA unit determine if the sponsor was compliant with the GLP regulations under 21 US Code of Federal Regulations 58. It is now unclear if there is an expectation that the CRO would need to perform an external audit to assure compliance. Attendees suggested considering including a compliance exception in the QA statement.

- Freeze-thaw stability: two findings were related to freeze-thaw stability. First, freeze-thaw stability of incurred sample reanalysis (ISR) samples exceeded the validated number of freeze-thaw cycles. Attendees agreed that freeze-thaw of these samples is inherently supported by the acceptability of the ISR results and no additional validation testing is required. Second, the thaw time during the evaluation was not representative of the time that study samples were thawed. It is suggested to thaw validation samples at least $2 \mathrm{~h}$ between cycles.

- Training SOPs: proficiency training on SOPs was not included in the training program. A process for proficiency training on SOPs is suggested. 
- Run interruptions: regulatory auditors cited that runs should not be interrupted for interim analysis of run acceptance, nor can assumptions be made regarding run acceptance prior to completion of the run. It is recommended to ensure that SOPs do not allow for interim analysis of run acceptance.

- Sponsor communication: regulatory auditors cited that correspondence and decisions for all events need to be documented and maintained within the study. It is suggested that SOPs outline the requirements for capturing, retaining and filing significant communications (including verbal ones) and correspondence. Specifically, these should include significant decisions that impact or affect study conduct.

- Sample storage: the SOP was lacking in the description for quarantine procedures and allowed for the use of open access storage units for temporary storage. It is suggested that SOPs outline the procedure for quarantining samples when they are received compromised. Additionally, the use of open access storage units is not recommended.

- Instrumentation SOPs: some instrumentation SOPs are not harmonized with user manual specifications and recommendations. It is recommended to include references to user manuals in applicable SOPs.

- Freezer mapping: regulatory auditors cited that temperature mapping of all storage units must be conducted because the results of this mapping must then be used to determine proper temperature probe placement.

- Time stamping documentation: regulatory auditors cited that time stamping is required on critical documentation to demonstrate that approvals of activities are conducted prior to their execution. It is suggested that critical forms be reformatted to include the requirement for recording the time of each dated signature.

\section{Electronic laboratory notebooks \& LIMS systems}

The topic of electronic laboratory notebooks (ELNs) was revisited again at this closed forum after being discussed during the 7th GCC meeting [5]. At that time, the CRO community was divided on the use of ELNs. Most member companies were still using paper, and although users of these systems saw benefits to quality and documentation, cost and a variety of types of studies causing adaptability issues were seen as deterrents to implementation. In order to determine if there had been a change to this position after two more years of experience, a survey was sent to member companies. Results indicate that only a third of respondents use an ELN at this time.

Queries into additional electronic systems were also asked. Most GCC members do not use electronic archiving software, but elect to archive electronic data manually. Thermo Watson LIMS ${ }^{\mathrm{TM}}$ is used by a majority of respondents, or a custom/in-house developed system. If Watson is used, it is principally for routine analysis or method validation, automated reassay selection and sample tracking.

\section{Future perspective}

The GCC will continue to provide recommendations on hot topics of global interest in small and large molecule bioanalysis, biomarkers and immunogenicity, and expand its membership by coordinating its activities with the regional and international meetings held by the pharmaceutical industry. Please contact the GCC [25] for the exact date and time of future meetings, and for all membership information.

\section{Acknowledgements}

The GCC would like to thank the following: Roger Hayes (MPI Research) for chairing the 9th GCC Closed Forum; Mario Rocci (ICON Laboratories) and Isabelle Dumont (Algorithme Pharma) for designing the survey, collecting answers, preparing survey results and chairing the sessions on 'The place of CAPA in regulated bioanalysis' and 'Method robustness in regulated bioanalysis'; Rafiq Islam (Celerion), Dominique Gouty (BioAgilytix) and Chris Beaver (inVentiv Health Clinical) for designing the survey, collecting answers, preparing survey results and chairing the session on 'Best practices for supporting biosimilar studies'; Richard LeLacheur (Agilux Labs), Elizabeth Groeber (WIL Research) and Colin Pattison (Charles River Labs) for designing the survey, collecting answers, preparing survey results and chairing the session on 'Status of method validation requirements in the preclinical space'; Afshin Safavi (BioAgilytix), John Kamerud (Eurofins Pharma Bioanalytics Services) and Laura Cojocaru (Tandem Labs-now Covance) for designing the survey, collecting answers, preparing survey results and chairing the session on 'Best practices for measuring endogenous biomarkers beyond prior GCC recommendations'; Philippe Couerbe (Atlanbio) and Stephanie Cape (Covance) for chairing the session on 'Whole blood stability and blood partitioning'; Roger Hayes (MPI Research) and Chad Briscoe (PRA Health Sciences) for chairing the sessions on 'Regulatory audit experiences' and 'Electronic laboratory notebooks and LIMS systems'; All the GCC member company representatives who filled in the numerous surveys used to prepare the discussion of the 9th GCC Closed Forum; All the member company representatives who have sent comments and suggestions to complete this commentary; Natasha Savoie (GCC) for taking the minutes of the 9th GCC Closed 
Forum and drafting the first version of this commentary; Wei Garofolo (GCC) for organizing the logistics of the meeting and coordinating the review of this commentary.

\section{Financial \& competing interests disclosure}

The authors have no relevant affiliations or financial involvement with any organization or entity with a financial

\section{Author affiliations}

'MPI Research, Mattawan, MI, USA

${ }^{2}$ Agilux Laboratories, Worcester, MA, USA

${ }^{3}$ Algorithme Pharma, Laval, QC, Canada

${ }^{4}$ Atlanbio, Saint-Nazaire, France

${ }^{5}$ BioAgilytix, Durham, NC, USA

${ }^{6}$ Celerion, Lincoln, NB, USA

${ }^{7}$ Charles River, Edinburgh, UK

${ }^{8}$ Covance, Madison, WI, USA

ICON, Whitesboro, NY, USA

${ }^{10}$ PRA Health Sciences, Lenexa, KS, USA

"Tandem Labs, West Trenton, NJ, USA, currently at Covance, WestTrenton, NJ, USA

${ }^{12}$ WIL Research, Ashland, OH, USA

${ }^{13} 3 \mathrm{~S}$-Pharmacological Cons. \& Res. GmbH, Harpstedt, Germany

${ }^{14} \mathrm{AlT}$ Bioscience Indianapolis, IN, USA

${ }^{15}$ Alturas Analytics, Moscow, ID, USA

${ }^{16}$ Anapharm Europe, Barcelona, Spain

${ }^{17}$ Axis Clinicals, Dilworth, MN, USA

${ }^{18}$ BASi, West Lafayette, IN, USA

${ }^{19}$ Bioanalytical Laboratory Services, Toronto, ON, Canada

${ }^{20}$ Biotrial Bioanalytical Services Inc., Laval, QC, Canada

${ }^{21}$ CAPRION, Montreal, QC, Canada

${ }^{22}$ Charles River, Senneville, QC, CAN, currently at Algorithme Pharma, Laval, QC, Canada

${ }^{23}$ Charles River, Reno, NV, USA

${ }^{24}$ CIRION BioPharma Research, Laval, QC, Canada

${ }^{25}$ Covance, Chantilly, VA, USA

\section{References}

1 Premkumar N, Lowes S, Jersey J et al. Formation of a global contract research organization council for bioanalysis. Bioanalysis 2(11), 1797-1800 (2010).

2 Breda N, Garofolo F, Cruz Caturla M et al. The 3rd Global $\mathrm{CRO}$ council for bioanalysis at the international reid bioanalytical forum. Bioanalysis 3(24), 2721-2727 (2011).

3 Lowes S, Jersey J, Shoup R et al. 4th Global CRO Council for Bioanalysis: coadministered drugs stability, EMA/US FDA Guidelines, 483s and Carryover. Bioanalysis 4(7), 763-768 (2012).

4 Nicholson R, Lowes S, Cruz Caturla M et al. 6th GCC focus on LBA: critical reagents, positive controls and reference standards; specificity for endogenous compounds; biomarkers; biosimilars. Bioanalysis 4(19), 2335-2342 (2012).

5 Rocci M, Lowes S, Shoup R et al. 7th GCC: use of incurred samples; fit-for-purpose validation; solution stability; electronic lab notebooks; hyperlipidemic matrix testing. Bioanalysis 6(20), 2713-2720 (2014).

6 Bower J, Fast D, Garofolo F et al. 8th GCC: consolidated feedback to US FDA on the 2013 Draft FDA Guidance interest in or financial conflict with the subject matter or materials discussed in the manuscript. This includes employment, consultancies, honoraria, stock ownership or options, expert testimony, grants or patents received or pending, or royalties.

No writing assistance was utilized in the production of this manuscript.

${ }^{26}$ Eurofins Pharma Bioanalytics Services, St Charles, MO, USA

${ }^{27}$ Frontage Laboratories, Inc., Exton, PA, USA

${ }^{28} \mathrm{Global}$ CRO Council for Bioanalysis (GCC)

${ }^{29} \mathrm{Global}$ CRO Council for Bioanalysis, Envigo, East Millstone, NJ, USA

${ }^{30}$ Intertek USA, San Diego, CA, USA

${ }^{31}$ Previously at InVentiv Health Clinical, Quebec City, QC, CAN

${ }^{32} \mathrm{CMIC}$, Inc., Hoffman Estates, IL, USA

${ }^{33}$ KCAS Bioanalytical Services, Shawnee, KS, USA, currently at Alliance Pharma, Malvern, PA, USA

${ }^{34} \mathrm{KCAS}$ Bioanalytical Services, Shawnee, KS, USA

${ }^{35}$ Keystone Bioanalytical, Inc., North Wales, PA, USA

${ }^{36}$ Lambda Therapeutic Research, Ahmedabad, India

${ }^{37} \mathrm{LGC}$, Fordham, UK

${ }^{38}$ Mercodia AB, Uppsala, Sweden

${ }^{39} \mathrm{PPD}$, Richmond, VA, USA

40PRA Health Sciences, Assen, The Netherlands

${ }^{41}$ Pyxant Labs, Colorado Springs, CO, USA

${ }^{42}$ Pharma Medica, Toronto, ON, Canada

${ }^{43}$ QPS, Groningen, The Netherlands, currently at Antaeus Biopharma, Groningen, Netherlands

${ }^{44}$ Quintiles, Ithaca, NY, USA

${ }^{45}$ Quintiles, Oss, The Netherlands

${ }^{46}$ Tandem Labs, Durham, NC, USA, currently at Covance, Durham,NC, USA

${ }^{47}$ Worldwide Clinical Trials, Austin, TX, USA

${ }^{48} \mathrm{WuXi} / \mathrm{XBL}, 107$ Morgan Lane, Plainsboro, NJ, USA

on Bioanalytical Method Validation. Bioanalysis 6(22), 2957-2963 (2014).

7 Lowes S, Jersey J, Shoup R et al. Recommendations on: Internal Standard Criteria, Stability, Incurred Sample Reanalysis and Recent 483s by the Global CRO Council for Bioanalysis. Bioanalysis 3(12), 1323-1332 (2011).

8 Boterman M, Doig M, Breda M et al. Recommendations on the interpretation of the new EMA Guideline on Bioanalytical Method Validation by Global CRO Council for Bioanalysis (GCC). Bioanalysis 4(6), 651-660 (2012).

9 Sangster T, Maltas J, Struwe P et al. Recommendations on ISR in multi-analyte assays, QA/bioanalytical consultants and GCP by Global CRO Council for Bioanalysis (GCC). Bioanalysis 4(14), 1723-1730 (2012).

10 Lowes S, Boterman M, Doig M et al. Recommendations on bioanalytical method stability implications of coadministered and co-formulated drugs by Global CRO Council for Bioanalysis (GCC). Bioanalysis 4(17), 2117-2126 (2012).

11 Hougton R, Gouty D, Allinson J et al. Recommendations on biomarker bioanalytical method validation by GCC. Bioanalysis 4(20), 2439-2446 (2012). 
12 Lowes S, LeLacheur R, Shoup R et al. Recommendations on Incurred Sample Stability (ISS) by GCC. Bioanalysis 6(18), 2385-2390 (2014).

13 US Department of Health and Human Services, US FDA, Center for drug evaluation and research, center for biologics evaluation and research. guidance for industry: Q10 pharmaceutical quality system (2009). www.fda.gov/downloads/Drugs/.../Guidances/ucm073517.pdf ICH Harmonised tripartite guideline: Q2(R1) validation of analytical procedures: text and methodology (1996). www.ich.org/fileadmin/Public_Web_Site/ICH_Products/ Guidelines/Quality/Q2_R1/Step4/Q2_R1__Guideline.pdf Center for drug evaluation and research, center for biologics evaluation and research. guidance for industry: analytical procedures and methods validation for drugs and biologics (2015).

www.fda.gov/downloads/drugs/

guidancecomplianceregulatoryinformation/guidances/ ucm386366.pdf

US Department of Health and Human Services, US FDA, Center for Drug Evaluation and Research, Center for Veterinary Medicine. Draft Guidance for Industry, Bioanalytical Method Validation, Rockville, MD USA (2013). www.fda.gov/downloads/drugs/ guidancecomplianceregulatoryinformation/guidances/ ucm368107.pdf

17 US Department of Health and Human Services, US FDA, Center for Drug Evaluation and Research, Center for Veterinary Medicine. Guidance for Industry, Bioanalytical Method Validation, Rockville, MD USA (2001). www.fda.gov/downloads/Drugs/Guidance/ucm070107.pdf

European Medicines Agency. Committee for Medicinal Products for Human Use (CHMP). Guideline on Bioanalytical Method Validation. London, UK (2011). EMEA/CHMP/EWP/192217/2009. www.ema.europa.eu/docs/en_GB/document_library/ Scientific_guideline/2011/08/WC500109686.pdf
19 ANVISA. Resolution RDC no 27, May 17, 2012. Brazilian Health Surveillance Agency, Brazil (2012).

http://bcn2012.europeanbioanalysisforum.eu/slides/day $\% 20$ 3/7\%20updates $\% 20$ from $\% 20$ the $\% 20$ globe/3_tavares.pdf

20 European Medicines Agency. Committee for Medicinal Products for Human Use (CHMP). Guideline on similar biological medicinal products. London, UK (2014). CHMP/437/04 Rev 1 www.ema.europa.eu/docs/en_GB/document_library/ Scientific_guideline/2009/09/WC500003517.pdf

21 US Department of Health and Human Services, US FDA, Center for Drug Evaluation and Research, Center for Biologics Evaluation and Research. Scientific Considerations in Demonstrating Biosimilarity to a Reference Product (2015). www.fda.gov/downloads/ DrugsGuidanceComplianceRegulatoryInformation/ Guidances/UCM291128.pdf

22 European Medicines Agency. Committee for Medicinal Products for Human Use (CHMP). Draft Reflection paper on guidance for laboratories that perform the analysis or evaluation of clinical trial samples. London, UK (2010). EMA/ INS/GCP/532137/2010

www.ema.europa.eu/docs/en_GB/document_library/ Regulatory_and_procedural_guideline/2010/09/ WC500096987.pdf

23 Houghton R, Gouty D Allinson J et al. Recommendations on biomarker bioanalytical method validation by GCC. Bioanalysis, 4(20), 2439-2446 (2012).

24 Freisleben A, Brudny-Kloppel M, Mulder H et al. Blood stability testing: European Bioanalysis Forum view on current challenges for regulated bioanalysis. Bioanalysis 3(12), 1333-1336 (2011).

25 Global CRO Council for Bioanalysis. www.global-cro-council.org 